\title{
Context-Sensitive Equivalences for Non-Interference based Protocol Analysis *
}

\author{
Michele Bugliesi, Ambra Ceccato, and Sabina Rossi \\ Dipartimento di Informatica, Università Ca' Foscari di Venezia \\ via Torino 155, 30172 Venezia, Italy \\ \{bugliesi, ceccato, srossi\}@dsi.unive.it
}

\begin{abstract}
We develop new proof techniques, based on non-interference, for the analysis of safety and liveness properties of cryptographic protocols expressed as terms of the process algebra CryptoSPA. Our approach draws on new notions of behavioral equivalence, built on top of a context-sensitive labelled transition system, that allow us to characterize the behavior of a process in the presence of any attacker with a given initial knowledge. We demonstrate the effectiveness of the approach with an example of a protocol of fair exchange.
\end{abstract}

\section{Introduction}

Non-Interference has been advocated by various authors [1,9] as a powerful method for the analysis of cryptographic protocols. In [9], Focardi et al. propose a general schema for specifying security properties with a uniform and concise definition. The approach draws on earlier work by the same authors on characterizing information-flow security in terms of Non-Interference for the Security Process Algebra (SPA, for short). We briefly review the main ideas below.

SPA is a variant of CCS in which the set of actions is partitioned into two sets: $L$, for low, and $H$ for high. A Non-Interference property $\mathcal{P}$ for a process $E$ is expressed as follows:

$$
E \in \mathcal{P} \text { if } \forall \Pi \in \mathcal{E}_{H}:(E|| \Pi) \backslash H \approx_{\mathcal{P}} E \backslash H
$$

where $\mathcal{E}_{H}$ is the set of all high-level processes, $\approx_{\mathcal{P}}$ is an observation equivalence (parametric in $\mathcal{P}$ ), $\|$ is parallel composition, and $\backslash$ is restriction. The processes $E \backslash H$ and $(E|| \Pi) \backslash H$ represent the low-level views of $E$ and of $E \| \Pi$, respectively. The basic intuition is expressed by the slogan: "If no high-level process can change the low behavior, then no flow of information from high to low is possible".

In [9] this idea is refined to provide a general definition of security properties for cryptographic protocols described as terms of CryptoSPA, a process algebra that extends SPA with cryptographic primitives. Intuitively, the refinement amounts to viewing the participants to a protocol as low-level processes, while the high-level processes

\footnotetext{
* This work has been partially supported by the MIUR project "Modelli formali per la sicurezza (MEFISTO)" and the EU project IST-2001-32617 "Models and types for security in mobile distributed systems (MyThS)".
}

A. Lingas and B.J. Nilsson (EDS): FCT 2003, LNCS 2571, pp. 364-375, 2003.

(C) Springer-Verlag, Berlin Heildelberg 2003. 
represent the external attackers. Then, Non-Interference implies that the attackers have no way to change the low (honest) behavior of the protocol.

There are two problems that need to be addressed to formalize this idea. First, the intruder should be assumed to have complete control over the public components of the network. Consequently, any step in a protocol involving a public channel should be classified as a high-level action. However, since a protocol specification is usually entirely determined by the exchange of messages over public channels, a characterization like (1) becomes trivial, as $(E|| \Pi) \backslash H$ and $E \backslash H$ are simply the null processes. This is easily rectified by extending the protocol specification with low-level actions that are used to specify the desired security property.

A further problem arises from the formalization of the perfect cryptography assumption that is usually made in the analysis of the logical properties of cryptographic protocols. In [9] this assumption is expressed by making the definition of Non-Interference dependent on the initial knowledge of the attacker and on a deduction system by which the attacker may compute new information. The initial knowledge, noted $\phi$, includes private data (e.g., the enemy's private keys) as well as any piece of publicly available information, such as names of entities and public keys. Property (1) is thus reformulated for a protocol $P$ as follows:

$$
P \in \mathcal{P} \text { if } \forall \Pi \in \mathcal{E}_{H}^{\phi}:(P|| \Pi) \backslash H \approx_{\mathcal{P}} P \backslash H .
$$

where $\mathcal{E}_{H}^{\phi}$ is the set of the high-level processes $\Pi$ which can perform only actions using the public channel names and whose messages (those syntactically appearing in $\Pi$ ) can be deduced from $\phi$.

This framework is very general, and lends itself to the characterization of various security properties, obtained by instantiating the equivalence $\approx_{\mathcal{P}}$ in the schema above. Instead, it is less effective as a proof method, due to the universal quantification over the possible intruders $\Pi$ in the class $\mathcal{E}_{H}^{\phi}$. In [9], the problem is circumvented by analyzing the protocol in presence of the "hardest attacker". However, In [9] this characterization is proved correct only for the class of relationships $\approx_{\mathcal{P}}$ that are behavioral preorders on processes. In particular, the proof method is not applicable for equivalences based on bisimulation, and consequently, for the analysis of certain, branching time, liveness properties, such as fairness.

We partially rectify the problem by developing a technique which does not require us to exhibit an explicit attacker (nor, in particular, it requires the existence of a hardest attacker). Our approach draws on ideas from [4] to represent the attacker indirectly, in terms of a context-sensitive labelled transition system. The labelled transitions take the form $\phi \triangleright P \stackrel{a}{\longrightarrow} \phi^{\prime} \triangleright P^{\prime}$, where $\phi$ represents the context's knowledge prior to the transition, and $\phi^{\prime}$ is the new knowledge resulting from $P$ performing the action $a$. Building on this labelled transition system we provide quantification-free characterizations for different instantiations of (2), specifically when $\approx_{\mathcal{P}}$ is instantiated to trace equivalence, and to weak bisimulation equivalence. This allows us to apply our technique to the analysis of safety as well as liveness security properties. We demonstrate the latter with an example of a protocol of fair exchange.

The rest of the presentation proceeds as follows: Section 2 briefly reviews the process algebra CryptoSPA, Section 3 introduces context-sensitive labelled transition sys- 
tems, Section 4 gives characterizations for various security properties, Section 5 illustrates the example, and Section 6 draws some conclusions.

All the results presented in this paper are described and proved in [7].

\section{The CryptoSPA Language}

The Cryptographic Security Process Algebra (CryptoSPA, for short) [9] is an extension of SPA [8] with cryptographic primitives and constructs for value passing. The syntax is based on the following elements: a set $M$ of basic messages and a set $K$ of encryption keys with a function ${ }^{-1}: K \longrightarrow K$ such that $\left(k^{-1}\right)^{-1}=k$; a set $\mathcal{M}$, ranged over by $m$, of all messages, defined as the least set containing $M \cup K$ and closed under the deduction rules in Table 1 (more on this below); a set $\mathcal{C}$ of channels partitioned into two sets $H$ and $L$ of high and low channels, respectively; a function $M s g$ which maps every channel $c$ into the set of messages that can be sent and received on $c$ and such that $\operatorname{Msg}(c)=\operatorname{Msg}(\bar{c})$; a set $\mathcal{L}=\{c(m) \mid m \in M s g(c)\} \cup\{\bar{c} m \mid m \in M s g(c)\}$ of visible actions and the set $A c t=\mathcal{L} \cup\{\tau\}$ of all actions, ranged over by $a$, where $\tau$ is the internal (invisible) action; a function $\operatorname{chan}(a)$ which returns $c$ if $a$ is either $c(m)$ or $\bar{c} m$ and the special channel void when $a=\tau$; a set Const of constants. By an abuse of notation, we write $c(m), \bar{c} m \in H$ whenever $c, \bar{c} \in H$, and similarly for $L$.

The syntax of CryptoSPA terms (or processes) is defined as follows:

$$
\begin{aligned}
P::= & \mathbf{0}|c(x) . P| \bar{c} m . P|\tau . P| P+P|P||P| P \backslash C|P[f]| \\
& \left|A\left(m_{1}, \ldots, m_{n}\right)\right|\left[m=m^{\prime}\right] P ; P \mid\left[\left\langle m_{1} \ldots m_{n}\right\rangle \vdash_{\text {rule }} x\right] P ; P
\end{aligned}
$$

Both $c(x) . P$ and $\left[\left\langle m_{1} \ldots m_{n}\right\rangle \vdash_{\text {rule }} x\right] P ; P^{\prime}$ bind the variable $x$ in $P$. Constants are defined as: $A\left(x_{1}, \ldots, x_{n}\right) \stackrel{\text { def }}{=} P$, where $P$ is a CryptoSPA process that may contain no free variables except $x_{1}, \ldots, x_{n}$, which must be pairwise distinct.

$$
\begin{gathered}
\frac{m \quad m^{\prime}}{\left(m, m^{\prime}\right)}\left(\vdash_{\text {pair }}\right) \quad \frac{\left(m, m^{\prime}\right)}{m}\left(\vdash_{f s t}\right) \frac{\left(m, m^{\prime}\right)}{m^{\prime}}\left(\vdash_{\text {snd }}\right) \\
\frac{\frac{m k}{\{m\}_{k}}\left(\vdash_{\text {enc }}\right) \quad \frac{\{m\}_{k} k^{-1}}{m}\left(\vdash_{\text {dec }}\right)}{m}
\end{gathered}
$$

Table 1. Inference system for message manipulation where $m, m^{\prime} \in \mathcal{M}$ and $k, k^{-1} \in K$

Intuitively, $\mathbf{0}$ is the empty process; $c(x) . P$ waits for input $m$ on channel $c$, and then behaves as $P[m / x]$ (i.e., $P$ with all the occurrences of $x$ substituted by $m) ; \bar{c}(m) . P$ outputs $m$ on channel $c$ and continues as $P ; P_{1}+P_{2}$ represents the nondeterministic 
choice between $P_{1}$ and $P_{2} ; P_{1} \| P_{2}$ is parallel composition, where executions are interleaved, possibly synchronized on complementary input/output actions, producing an internal action $\tau ; P \backslash C$ is like $P$ but prevented from sending and receiving messages on channels in $C \subseteq C$; in $P[f]$ every channel $c$ is relabelled into $f(c) ; A\left(m_{1}, \ldots, m_{n}\right)$ behaves like the respective definition where the variables $x_{1}, \cdots, x_{n}$ are substituted with messages $m_{1}, \cdots, m_{n}$; $\left[m=m^{\prime}\right] P_{1} ; P_{2}$ behaves as $P_{1}$ if $m=m^{\prime}$ and as $P_{2}$ otherwise; finally, $\left[\left\langle m_{1} \ldots m_{n}\right\rangle \vdash_{\text {rule }} x\right] P_{1} ; P_{2}$ tries to deduce an information $z$ from the tuple $\left\langle m_{1} \ldots m_{n}\right\rangle$ through rule $\vdash_{\text {rule }}$; if it succeeds then it behaves as $P_{1}[z / x]$, otherwise it behaves as $P_{2}$.

In formalizing the security properties of interest, we will find it convenient to rely on (an equivalent of) the hiding operator, of CSP, noted $P / C$ with $P$ process and $C \subseteq$ $C$, which turns all actions using channels in $C$ into internal $\tau$ 's. This operator can be defined in CryptoSPA as follows: given any set $C \subseteq C, P / C \stackrel{\text { def }}{=} P\left[f_{C}\right]$ where $f_{C}(a)=$ $a$ if $\operatorname{chan}(a) \notin C$ and $f_{C}(a)=\tau$ if $\operatorname{chan}(a) \in C$.

We denote by $\mathcal{E}$ the set of all CryptoSPA processes and by $\mathcal{E}_{H}$ the set of all highlevel processes, i.e., those constructed only using actions in $H \cup\{\tau\}$.

The operational semantics of CryptoSPA is defined in terms of the labelled transition system (LTS) in Table 2. Most of the transitions are standard, and simply formalize the intuitive semantics of the process constructs discussed above. The two rules $\left(\vdash_{i}\right)$ connect the deduction system in in Table 1 with the transition system. The former system is used to model the ability of the attacker to deduce new information from its initial knowledge. Note, in particular, that secret keys, not initially known to the attacker, may not be deduced (hence we disregard cryptographic attacks, based on guessing secret keys). We say that $m$ is deducible from a set of messages $\phi$ (and write $\phi \vdash m$ ) if $m$ can be obtained from $\phi$ by applying the inference rules in Table 1. As in [9] we assume that $\vdash$ is decidable.

We complement the definition of the semantics with a corresponding notion of $o b$ servation equivalence, which is used to establish equalities among processes and is based on the idea that two systems have the same semantics if and only if they cannot be distinguished by an external observer. The equivalences that are relevant to the present discussion are trace equivalence, noted $\approx_{T}$, and weak bisimulation, noted $\approx_{B}$ (see [13]).

In the next section, we introduce coarser versions of these equivalences, noted $\approx_{T}^{\phi}$ and $\approx_{B}^{\phi}$, which distinguish processes in contexts with initial knowledge $\phi$. These context-sensitive notions of equivalence are built on a refined version of the labelled transition system, which we introduce next.

\section{Context-Sensitive Equivalences}

Following [4], we characterize the behavior of processes in terms of "context-sensitive labelled transitions" where each process transition depends on the knowledge of the context. To motivate, consider a process $P$ that produces and sends a message $\{m\}_{k}$ reaching the state $P^{\prime}$, and assume that $m$ and $k$ are known to $P$ but not to the context. Under these hypotheses, the context will never be able to reply the message $m$ to $P^{\prime}$ (or any continuation thereof). Hence, if $P^{\prime}$ waits for further input, we can safely leave 
any input transition involving $m$ out of the LTS, as the $P^{\prime}$ will never receive $m$ from the context.

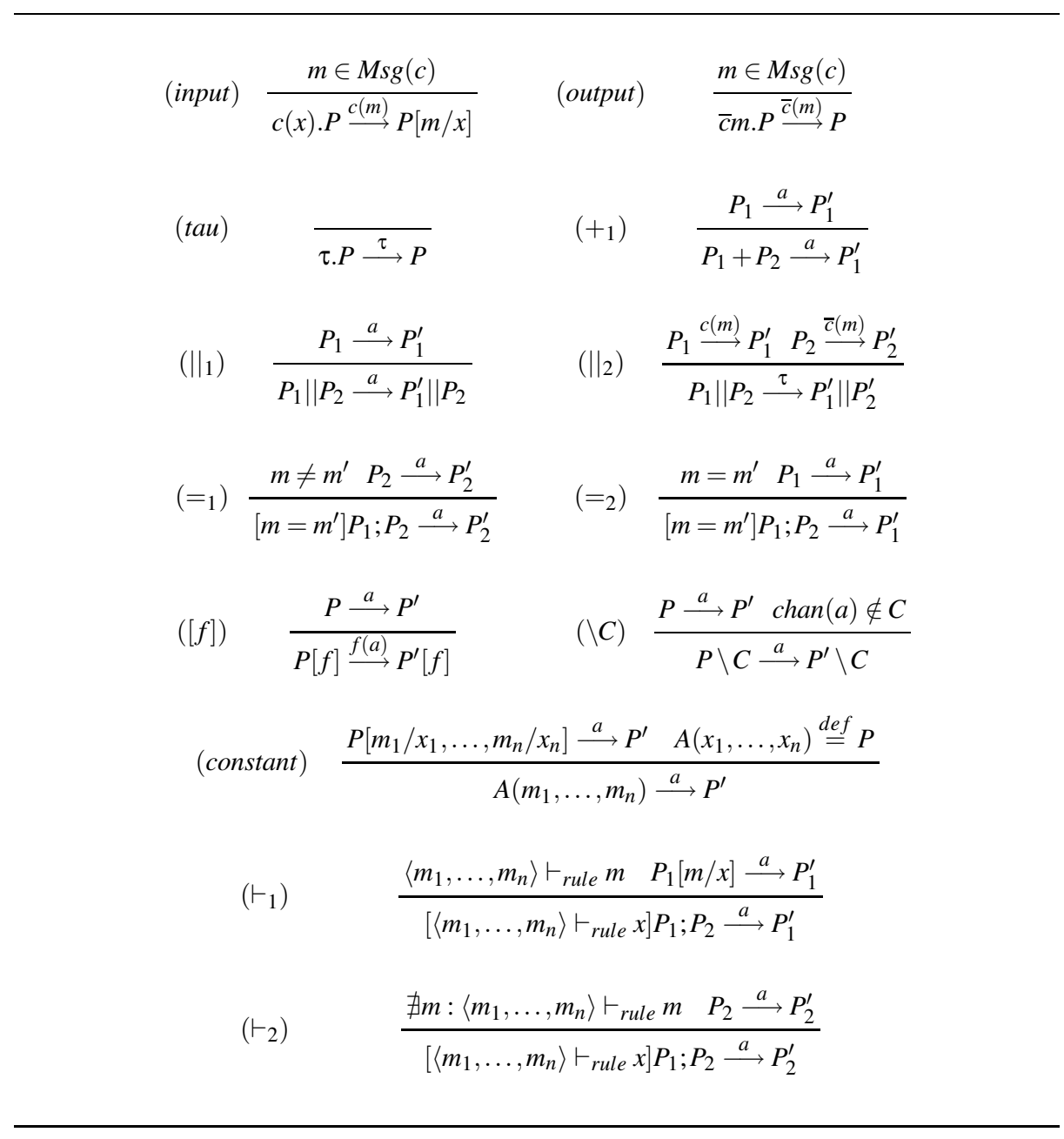

Table 2. The operational rules for CryptoSPA

The states of the new labelled transition system are configurations of the form $\phi \triangleright P$, where $P$ is a process and $\phi$ is the current knowledge of the context, represented through a set of messages. The transitions represent interactions between the process and the context and now take the form

$$
\phi \triangleright P \stackrel{a}{\longrightarrow} \phi^{\prime} \triangleright P^{\prime},
$$




$$
\begin{aligned}
& \text { (output) } \frac{P \stackrel{\bar{c} m}{\longrightarrow} P^{\prime} \quad \bar{c} m \in H}{\phi \triangleright P \stackrel{\bar{c}(m)}{\longrightarrow} \phi \cup\{m\} \triangleright P^{\prime}} \quad \text { (input) } \frac{P \stackrel{c(m)}{\longrightarrow} P^{\prime} \quad \phi \vdash m \quad c(m) \in H}{\phi \triangleright P \stackrel{c(m)}{\longrightarrow} \phi \triangleright P^{\prime}} \\
& \text { (tau) } \frac{P \stackrel{\tau}{\longrightarrow} P^{\prime}}{\phi \triangleright P \stackrel{\tau}{\longrightarrow} \phi \triangleright P^{\prime}} \quad \text { (low) } \quad \frac{P \stackrel{a}{\longrightarrow} P^{\prime} a \in L}{\phi \triangleright P \stackrel{a}{\longrightarrow} \phi \triangleright P^{\prime}}
\end{aligned}
$$

Table 3. Inference rules for the ELTS

where $a$ is the action executed by the process $P$ and $\phi^{\prime}$ is the new knowledge at disposal to the context for further interactions with $P^{\prime}$.

The transitions between configurations, in Table 3 , are defined rather directly starting from the corresponding transitions between processes. In rule (output), the context's knowledge is augmented with the information sent by the process. Dually, rule (input) assumes that the context performs an output action synchronizing with the input of the process. The message sent by the context must be completely deducible from the context's knowledge $\phi$, otherwise the corresponding transition is impossible: this is how the new transitions provide an explicit account of the attacker's knowledge. The remaining rules, (tau) and (low) state that internal actions of the protocol, and low actions do not contribute to the knowledge of the context in any way.

In the rest of the presentation, we refer to the transition rules in Table 3 collectively as the enriched LTS (ELTS, for short). Also, we assume that the initial knowledge of the context includes only public information and the context's private names. This is a reasonable condition, since it simply corresponds to assuming that each protocol run starts with fresh keys and nonces, a condition that is readily guaranteed by relying on time-dependent elements (e.g., time-stamps) and assuming that session keys are distinct for every executions.

The notions of trace and weak bisimulation equivalences extend in the expected way from processes to ELTS configurations, as we discuss below.

We write $\phi \triangleright P \stackrel{a}{\Longrightarrow} \phi^{\prime} \triangleright P^{\prime}$ to denote the sequence of transitions $\phi \triangleright P(\stackrel{\tau}{\longrightarrow})^{*} \phi \triangleright$ $P_{1} \stackrel{a}{\longrightarrow} \phi^{\prime} \triangleright P_{2}(\stackrel{\tau}{\longrightarrow})^{*} \phi^{\prime} \triangleright P^{\prime}$, where, as expected, $\phi=\phi^{\prime}$ if $\stackrel{a}{\longrightarrow}$ is an input, low or silent action. Furthermore, let $\gamma=a_{1} \ldots a_{n} \in \mathcal{L}^{*}$ be a sequence of (non silent) actions; then $\phi \triangleright P \stackrel{\gamma}{\Longrightarrow} \phi^{\prime} \triangleright P^{\prime}$ if there are $P_{1}, P_{2}, \ldots, P_{n-1} \in \mathcal{E}$ and $\phi_{1}, \phi_{2}, \ldots, \phi_{n-1}$ states such that $\phi \triangleright P \stackrel{a_{1}}{\Longrightarrow} \phi_{1} \triangleright P_{1} \stackrel{a_{2}}{\Longrightarrow} \ldots \stackrel{a_{n-1}}{\Longrightarrow} \phi_{n-1} \triangleright P_{n-1} \stackrel{a_{n}}{\Longrightarrow} \phi^{\prime} \triangleright P^{\prime}$. The notation $\phi \triangleright P \stackrel{\hat{a}}{\Longrightarrow} \phi^{\prime} \triangleright P^{\prime}$ stands for $\phi \triangleright P \stackrel{a}{\Longrightarrow} \phi^{\prime} \triangleright P^{\prime}$ if $a \in \mathcal{L}$ and for $\phi \triangleright P(\stackrel{\tau}{\longrightarrow})^{*} \phi \triangleright P^{\prime}$ if $a=\tau$, as usual.

\section{Definition 1 (Trace Equivalence over configurations).}

- $T(\phi \triangleright P)=\left\{\gamma \in \mathcal{L}^{*} \mid \exists \phi^{\prime}, P^{\prime}: \phi \triangleright P \stackrel{\gamma}{\Longrightarrow} \phi^{\prime} \triangleright P^{\prime}\right\}$ is the set of traces associated with the configuration $\phi \triangleright P$. 
- Two configurations $\phi_{P} \triangleright P$ and $\phi_{Q} \triangleright Q$ are trace equivalent, denoted by $\phi_{P} \triangleright P \approx_{T}^{c}$ $\phi_{Q} \triangleright Q$, if $T\left(\phi_{P} \triangleright P\right)=T\left(\phi_{Q} \triangleright Q\right)$.

Based on trace equivalence over configurations we can then define a corresponding notion of process equivalence, for processes executing in an environment with initial knowledge $\phi$. Formally, $P \approx_{T}^{\phi} Q$ whenever $\phi \triangleright P \approx_{T}^{c} \phi \triangleright Q$.

Definition 2 (Weak Bisimulation over configurations).

- A binary relation $\mathcal{R}$ over configurations is a weak bisimulation if, assuming $\left(\phi_{P} \triangleright\right.$ $\left.P, \phi_{Q} \triangleright Q\right) \in \mathcal{R}$, one has, for all $a \in A c t$ :

- if $\phi_{P} \triangleright P \stackrel{a}{\longrightarrow} \phi_{P^{\prime}} \triangleright P^{\prime}$, then there exists a configuration $\phi_{Q^{\prime}} \triangleright Q^{\prime}$ such that $\phi_{Q} \triangleright Q \stackrel{\hat{a}}{\Longrightarrow} \phi_{Q^{\prime}} \triangleright Q^{\prime}$ and $\left(\phi_{P^{\prime}} \triangleright P^{\prime}, \phi_{Q^{\prime}} \triangleright Q^{\prime}\right) \in \mathcal{R}$;

- if $\phi_{Q} \triangleright Q \stackrel{a}{\longrightarrow} \phi_{Q^{\prime}} \triangleright Q^{\prime}$, then there exists a configuration $\phi_{P^{\prime}} \triangleright P^{\prime}$ such that $\phi_{P} \triangleright P \stackrel{\hat{a}}{\Longrightarrow} \phi_{P^{\prime}} \triangleright P^{\prime}$ and $\left(\phi_{P^{\prime}} \triangleright P^{\prime}, \phi_{Q^{\prime}} \triangleright Q^{\prime}\right) \in \mathcal{R}$.

- Two configurations $\phi_{P} \triangleright P$ and $\phi_{Q} \triangleright Q$ are weakly bisimilar, denoted by $\phi_{P} \triangleright P \approx_{B}^{c}$ $\phi_{Q} \triangleright Q$, if there exists a weak bisimulation containing the pair $\left(\phi_{P} \triangleright P, \phi_{Q} \triangleright Q\right)$.

It is not difficult to prove that relation $\approx_{B}^{c}$ is the largest weak bisimulation over configurations, and that it is an equivalence relation. As for trace equivalence, we can recover an equivalence relation on processes executing in a context with initial knowledge $\phi$ by defining $P \approx_{B}^{\phi} Q$ if and only if $\phi \triangleright P \approx_{B}^{c} \phi \triangleright Q$.

\section{Non-Interference Proof Techniques}

We show that the new definitions of behavioral equivalence may be used to construct effective proof methods for various security properties within the general schema proposed in [9]. In particular, we show that making our equivalences dependent on the initial knowledge of the attacker provides us with security characterizations that are stated independently from the attacker itself.

The first property we study, known as NDC, results from instantiating $\approx_{\mathcal{P}}$ in (2) (see the introduction) to the trace equivalence relation $\approx_{T}$. As discussed in [9], NDC is a generalization of the classical idea of Non-Interference to non-deterministic systems and can be used for analyzing different security properties of cryptographic protocols such as secrecy, authentication and integrity. NDC can readily be extended to account for the context's knowledge as follows:

Definition 3 (NDC $\left.^{\phi}\right) . P \in N D C^{\phi}$ if $P \backslash H \approx_{T}(P \| \Pi) \backslash H, \forall \Pi \in \mathcal{E}_{H}^{\phi}$.

A process $P$ is $N D C^{\phi}$ if for every high-level process $\Pi$ with initial knowledge $\phi$ a low level user cannot distinguish $P$ from $(P \| \Pi)$, i.e., if $\Pi$ cannot interfere with the low-level execution of the process $P$.

Focardi et al. in [9] show that when $\phi$ is finite it is possible to find a most general intruder $T o p^{\phi}$ so that verifying $N D C^{\phi}$ reduces to checking $P \backslash H \approx_{T}\left(P|| T o p^{\phi}\right) \backslash H$. Here we provide an alternative ${ }^{1}$, quantification-free characterization of $N D C^{\phi}$. Let $P / H$ denote the process resulting from $P$, by replacing all high-level actions with the silent action $\tau$ (cf. Section 2). 
Theorem $1\left(\right.$ NDC $\left.^{\phi}\right) . P \in N D C^{\phi}$ if and only if $P \backslash H \approx_{T}^{\phi} P / H$.

More interestingly, our approach allows us to find a sound proof method for the $B N D C^{\phi}$ property, which results from instantiating (2) in the introduction with the equivalence $\approx_{B}$ as follows:

Definition 4 (BNDC $^{\phi}$ ). $P \in B N D C^{\phi}$ if $P \backslash H \approx_{B}(P|| \Pi) \backslash H, \forall \Pi \in \mathcal{E}_{H}^{\phi}$.

As for $N D C^{\phi}$, the definition falls short of providing a proof method due to the universal quantification over $\Pi$. Here, however, the problem may not be circumvented by resorting to a hardest attacker, as the latter does not exist, being there no (known) preorder on processes corresponding to weak bisimilarity.

What we propose here is a partial solution that relies on providing a coinductive (and quantification free) characterization of a sound approximation of $B N D C^{\phi}$, based on the following persistent version of $B N D C^{\phi}$.

Definition 5 (P_BNDC $\left.{ }^{\phi}\right) . P \in P \_B N D C^{\phi}$ if $P^{\prime} \in B N D C^{\phi}, \forall P^{\prime}$ reachable from $P$.

$P \_B N D C^{\phi}$ is the context-sensitive version of the $P \_B N D C$ property studied in [10]. Following the technique in [10], one can show that $P B N D C^{\phi}$ is a sound approximation of $B N D C^{\phi}$ which admits elegant quantification-free characterizations. Specifically, like $P \_B N D C, P \_B N D C^{\phi}$ can be characterized both in terms of a suitable weak bisimulation relation "up to high-level actions", noted $\approx_{\backslash H}^{\phi}$, and in terms of unwinding conditions, as discussed next. We first need the following definition:

Definition 6. Let $a \in$ Act. The transition relation $\stackrel{\hat{a}}{\Longrightarrow} \backslash H$ is defined as follows:

$$
\stackrel{\hat{a}}{\Longrightarrow} \backslash H= \begin{cases}\stackrel{\hat{a}}{\Longrightarrow} & \text { if } a \notin H \\ \stackrel{a}{\Longrightarrow} \text { or } \stackrel{\hat{\tau}}{\Longrightarrow} & \text { if } a \in H\end{cases}
$$

The transition relation $\stackrel{\hat{a}}{\Longrightarrow} \backslash H$ is defined as $\stackrel{\hat{a}}{\Longrightarrow}$, except that it treats $H$-level actions as silent actions. Now, weak bisimulations up to $H$ over configurations are defined as weak bisimulations over configurations except that they allow a high action to be matched by zero or more high actions. Formally:

\section{Definition 7 (Weak Bisimulation up to $H$ over configurations).}

- A binary relation $\mathcal{R}$ over configurations is a weak bisimulation up to $H$ if ( $\phi_{P} \triangleright$ $\left.P, \phi_{Q} \triangleright Q\right) \in \mathcal{R}$ implies that, for all $a \in A c t$,

- if $\phi_{P} \triangleright P \stackrel{a}{\longrightarrow} \phi_{P^{\prime}} \triangleright P^{\prime}$, then there exists a configuration $\phi_{Q^{\prime}} \triangleright Q^{\prime}$ such that $\phi_{Q} \triangleright Q \stackrel{\hat{a}}{\Longrightarrow} \backslash H \phi_{Q^{\prime}} \triangleright Q^{\prime}$ and $\left(\phi_{P^{\prime}} \triangleright P^{\prime}, \phi_{Q^{\prime}} \triangleright Q^{\prime}\right) \in \mathcal{R}$;

- if $\phi_{Q} \triangleright Q \stackrel{a}{\longrightarrow} \phi_{Q^{\prime}} \triangleright Q^{\prime}$, then there exists a configuration $\phi_{P^{\prime}} \triangleright P^{\prime}$ such that $\phi_{P} \triangleright P \stackrel{\hat{a}}{\Longrightarrow} \backslash H \phi_{P^{\prime}} \triangleright P^{\prime}$ and $\left(\phi_{P^{\prime}} \triangleright P^{\prime}, \phi_{Q^{\prime}} \triangleright Q^{\prime}\right) \in \mathcal{R}$.

\footnotetext{
${ }^{1}$ An analogous result has been recently presented by Gorrieri et al. in [11] for a timed extension of CryptoSPA. We discuss the relationships between our and their result in Section 6.
} 
- Two configurations $\phi_{P} \triangleright P$ and $\phi_{Q} \triangleright Q$ are weakly bisimilar up to $H$, denoted by $\phi_{P} \triangleright P \approx{ }_{\backslash H}^{c} \phi_{Q} \triangleright Q$, if there exists a weak bisimulation up to $H$ containing the pair $\left(\phi_{P} \triangleright P, \phi_{Q} \triangleright Q\right)$.

Again, we can prove that the relation $\approx_{\backslash H}^{c}$ is the largest weak bisimulation up to $H$ over configurations and that it is an equivalence relation. Also, as for previous relations over configurations, we can recover an associated relation over processes in a context with initial knowledge $\phi$ by defining

$$
P \approx_{\backslash H}^{\phi} Q \text { if and only if } \phi \triangleright P \approx_{\backslash H}^{c} \phi \triangleright Q .
$$

We can finally state the two characterizations of $P \_B N D C^{\phi}$. The former characterization is expressed in terms of $\approx_{j H}^{\phi}$ (with no quantification on the reachable states and on the high-level malicious processes).

Theorem 2 (P_BNDC ${ }^{\phi}$ 1). $P \in P \_B N D C^{\phi}$ if and only if $P \backslash H \approx_{\backslash H}^{\phi} P$.

The second characterization of $P B N D C^{\phi}$ is given in terms of unwinding conditions which demand properties of individual actions. Unwinding conditions aim at "distilling" the local effect of performing high-level actions and are useful to define both proof systems (see, e.g., [6]) and refinement operators that preserve security properties, as done in [12].

Theorem 3 (P_BNDC ${ }^{\phi}$ 2). $P \in P \_B N D C^{\phi}$ if and only if for all $\phi_{i} \triangleright P_{i}$ reachable from $\phi \triangleright P$, if $\phi_{i} \triangleright P_{i} \stackrel{h}{\longrightarrow} \phi_{i}^{\prime} \triangleright P_{i}^{\prime}$ for $h \in H$, then $\phi_{i} \triangleright P_{i} \stackrel{\hat{\tau}}{\longrightarrow} \phi_{i}^{\prime \prime} \triangleright P_{i}^{\prime \prime}$ such that $\phi_{i}^{\prime} \triangleright P_{i}^{\prime} \backslash H \approx_{B}^{c}$ $\phi_{i}^{\prime \prime} \triangleright P_{i}^{\prime \prime} \backslash H$.

Both the characterizations can be used for verifying cryptographic protocols. A concrete example of a fair exchange protocol is illustrated in the next section.

\section{An Example: the ASW fair exchange protocol}

The ASW contract signing protocol [2] is used in electronic commerce transactions to enable two parties, named $O$ (originator) and $R$ (responder), to obtain each other's commitment on a previously agreed contractual text $M$. To deal with unfair situations, each party may appeal to a trusted third party $T$ which can decide, on the basis of the data it has received, whether to issue a replacement contract or an abort token. If both $O$ and $R$ are honest, and they receive the messages sent to them, then they both obtain a valid contract upon the completion of the protocol.

We say that the protocol guarantees fairness to $O$ (dually, to $R$ ) on message $M$, if whatever malicious $R(O)$ is considered, if $R(O)$ gets evidence that $O(R)$ has originated $M$ then also $O(R)$ will eventually obtain the evidence that $R(O)$ has received $M$. Notice that this is a branching-time liveness property: we are requiring that something should happen if $O$ (resp. $R$ ) gets his evidence -i.e., that also $R$ (resp. $O$ ) should get his evidence - for all the execution traces in the protocol (cf. [9] for a thorough discussion on this point). 
The protocol consists of three independent sub-protocols: exchange, abort and resolve. Here, we focus on the main exchange sub-protocol that is specified by the following four messages, where $M$ is the contractual text on which we assume the two parties previously agreed, while $S K_{O}$ and $S K_{R}\left(P K_{O}\right.$ and $\left.P K_{R}\right)$ are the private (public) keys of $O$ and $R$, respectively.

$$
\begin{aligned}
& O \rightarrow R: m e_{1}=\left\{M, h\left(N_{O}\right)\right\}_{S K_{O}} \\
& R \rightarrow O: m e_{2}=\left\{\left\{M, h\left(N_{O}\right)\right\}_{S K_{O}}, h\left(N_{R}\right)\right\}_{S K_{R}} \\
& O \rightarrow R: m e_{3}=N_{O} \\
& R \rightarrow O: e_{4}=N_{R}
\end{aligned}
$$

In the first step, $O$ commits to the contractual text by hashing a random number $N_{O}$, and signing a message that contains both $h\left(N_{O}\right)$ and $M$. While $O$ does not actually reveal the value of its contract authenticator $N_{O}$ to the recipient of message $m e_{1}, O$ is committed to it. As in a standard commitment protocol, we assume that it is not computationally feasible for $O$ to find a different number $N_{O}^{\prime}$ such that $h\left(N_{O}^{\prime}\right)=h\left(N_{O}\right)$. In the second step, $R$ replies with its own commitment. Finally, $O$ and $R$ exchange the actual contract authenticators.

We specify the sub-protocol in CryptoSPA (see the figure below), by introducing some low-level actions to verify the correctness of protocol's executions. We say that an execution is correct if we observe the sequence of low-level actions $\overline{\text { received }} \mathrm{me}_{1}$, $\overline{\text { received }} \_e_{2}, \overline{\text { received }} N_{O}$, $\overline{\text { received }} N_{R}$ in this order.

$$
\begin{aligned}
& O\left(M, N_{O}\right) \stackrel{\text { def }}{=}\left[\left\langle N_{O}, k_{h}\right\rangle \vdash_{e n c} n\right]\left[\left\langle(M, n), S K_{O}\right\rangle \vdash_{e n c} p\right] \bar{c} p . c(v) . \\
& {\left[\left\langle v, P K_{R}\right\rangle \vdash_{\text {dec }} i\right]\left[i \vdash_{f_{s t}} p^{\prime}\right]\left[i \vdash_{\text {snd }} r^{\prime}\right]\left[p^{\prime}=p\right] \text { received_ov }_{-} .} \\
& \bar{c} N_{O} . c(j) .\left[\left\langle j, k_{h}\right\rangle \vdash_{\text {enc }} r^{\prime \prime}\right]\left[r^{\prime \prime}=r^{\prime}\right] \overline{\text { received }}_{-} j \\
& R\left(M, N_{R}\right) \stackrel{\text { def }}{=} c(q) .\left[\left\langle q, P K_{O}\right\rangle \vdash_{\text {dec }} s\right]\left[s \vdash_{f s t} m\right]\left[s \vdash_{\text {snd }} n^{\prime}\right][m=M] \overline{\text { received_q }} q . \\
& {\left[\left\langle N_{R}, k_{h}\right\rangle \vdash_{e n c} r\right]\left[\left\langle(q, r), S K_{R}\right\rangle \vdash_{e n c} t\right] \bar{c} t . c(u) .} \\
& {\left[\left\langle u, k_{h}\right\rangle \vdash \vdash_{\text {enc }} n^{\prime \prime}\right]\left[n^{\prime \prime}=n^{\prime}\right] \overline{\text { received_ }} \text { u. } \bar{c} N_{R}} \\
& P \stackrel{\text { def }}{=} O\left(M, N_{O}\right) \| R\left(M, N_{R}\right)
\end{aligned}
$$

Fig. 1. The CryptoSPA specification of the exchange sub-protocol of ASW

We can demonstrate that the protocol does not satisfy property $P \_B N D C^{\phi}$ when $\phi$ consists of public information and private data of possible attacker's. This can be easily checked by applying Theorem 3 . Indeed, just observing the protocol ELTS, one can immediately notice that there exists a configuration transition $\phi \triangleright P \stackrel{a}{\longrightarrow} \phi^{\prime} \triangleright P^{\prime}$, where $a=\bar{c} m e_{1}$, but there isn't any $\phi^{\prime \prime}$ and $P^{\prime \prime}$ such that $\phi \triangleright P \stackrel{\hat{\tau}}{\Longrightarrow} \phi^{\prime \prime} \triangleright P^{\prime \prime}$ and $\phi^{\prime} \triangleright$ $P^{\prime} \backslash H \approx_{B}^{c} \phi_{i}^{\prime \prime} \triangleright P_{i}^{\prime \prime} \backslash H$. In fact, it is easy to prove that $\phi^{\prime} \triangleright P^{\prime} \backslash H \approx_{B}^{c} \mathbf{0}$ for all $\phi^{\prime}$, while $\phi^{\prime \prime} \triangleright P^{\prime \prime} \backslash H \not_{B}^{c} \mathbf{0}$ for all $P^{\prime \prime}$ and $\phi^{\prime \prime}$ such that $\phi \triangleright P \stackrel{\hat{\tau}}{\Longrightarrow} \phi^{\prime \prime} \triangleright P^{\prime \prime}$. However, the fact that, in this case, the ASW protocol does not satisfy $P \triangle B N D C^{\phi}$ does not represent a real attack to the protocol since such a situation is resolved by inching the trusted party $T$. 
More interestingly, we can analyze the protocol under the assumption that one of the participants is corrupt. This can be done by augmenting the knowledge $\phi$ with the corrupt party's private information such as its private key and its contract authenticator. We can show that the protocol does not satisfy $P_{-} B N D C^{\phi}$ when $O$ is corrupt, finding the attack already described in [14].

\section{Conclusions and Related Work}

We have studied context-sensitive equivalence relationships and relative proof techniques within the process algebra CryptoSPA to analyze protocols. Our approach builds on context-sensitive labelled transition systems, whose transitions are constrained by the knowledge of the environment. We showed that our technique can be used to analyze both safety and liveness properties of cryptographic protocols.

In a recent paper Gorrieri et al. [11] prove results related to ours, for a real-time extension of CryptoSPA. In particular, they prove an equivalent of Theorem 1: however, while the results are equivalent, the underlying proof techniques are not. More precisely, instead of using context-sensitive LTS's, [11] introduces a special hiding operator $/ \phi$ and prove that $P \in N D C^{\phi}$ if and only if $P \backslash H \approx_{T} P /{ }^{\phi} H$. Process $P /{ }^{\phi} H$ corresponds exactly to our configuration $\phi \triangleright P / H$, in that the corresponding LTS's are isomorphic. However, the approach of [11] is still restricted to the class of observation equivalences that are behavioral preorders on processes and thus it does not extend to bisimulations.

As we pointed out since the outset, our approach is inspired by Boreale, De Nicola and Pugliese's work [4] on characterizing may test and barbed congruence in the spi calculus by means of trace and bisimulation equivalences built on top of context-sensitive LTS's. Based on the same technique, symbolic semantics and compositional proofs have been recently studied in $[3,5]$, providing effective tools for the verification of cryptographic protocols. Symbolic description methods could be exploited to deal with the state-explosion problems which are intrinsic in the construction of context-sensitive labelled transition systems. Future plans include work in that direction.

\section{References}

1. M. Abadi. Security Protocols and Specifications. In W. Thomas, editor, Proc. of the Second International Conference on Foundations of Software Science and Computation Structure (FoSSaCS'99), volume 1578 of LNCS, pages 1-13. Springer-Verlag, 1999.

2. N. Asokan, V. Shoup, and M. Waidener. Asynchronuous Protocols for Optimistic Fair Exchange. In Proc. of the IEEE Symposium on Research in Security and Privacy, pages 86-99. IEEE Computer Society Press, 1998.

3. M. Boreale and M. G. Buscemi. A Framework for the Analysis of Security Protocols. In Proc. of the 13th International Conference on Concurrency Theory (CONCUR'02), volume 2421 of $L N C S$, pages 483-498. Springer-Verlag, 2002.

4. M. Boreale, R. De Nicola, and R. Pugliese. Proof Tecniques for Cryptographic Processes. In Proc. of the 14th IEEE Symposium on Logic in Computer Science (LICS'99), pages 157-166. IEEE Computer Society Press, 1999.

5. M. Boreale and D. Gorla. On Compositional Reasoning in the spi-calculus. In Proc. of the 5th International Conference on Foundations of Software Science and Computation Structures (FossaCS'02), volume 2303 of LNCS, pages 67-81. Springer-Verlag, 2002. 
6. A. Bossi, R. Focardi, C. Piazza, and S. Rossi. A Proof System for Information Flow Security. In M. Leuschel, editor, Proc. of Int. Workshop on Logic Based Program Development and Transformation, LNCS. Springer-Verlag, 2002. To appear.

7. A. Ceccato. Analisi di protocolli crittografici in contesti ostili. Laurea thesis, Università Ca' Foscari di Venezia, 2001.

8. R. Focardi and R. Gorrieri. Classification of Security Properties (Part I: Information Flow). In R. Focardi and R. Gorrieri, editors, Foundations of Security Analysis and Design, volume 2171 of LNCS. Springer-Verlag, 2001.

9. R. Focardi, R. Gorrieri, and F. Martinelli. Non Interference for the Analysis of Cryptographic Protocols. In U. Montanari, J.D.P. Rolim, and E. Welzl, editors, Proc. of Int. Colloquium on Automata, Languages and Programming (ICALP'O0), volume 1853 of LNCS, pages 744755. Springer-Verlag, 2000.

10. R. Focardi and S. Rossi. Information Flow Security in Dynamic Contexts. In Proc. of the 15th IEEE Computer Security Foundations Workshop, pages 307-319. IEEE Computer Society Press, 2002.

11. R. Gorrieri, E. Locatelli, and F. Martinelli. A Simple Language for Real-time Cryptographic Protocol Analysis. In Proc. of 12th European Symposium on Programming Languages and Systems, LNCS. Springer-Verlag, 2003. To appear.

12. H. Mantel. Unwinding Possibilistic Security Properties. In Proc. of the European Symposium on Research in Computer Security, volume 2895 of LNCS, pages 238-254. Springer-Verlag, 2000.

13. R. Milner. Communication and Concurrency. Prentice-Hall, 1989.

14. V. Shmatikov and J. C. Mitchell. Analysis of a Fair Exchange Protocol. In Proc. of 7th Annual Symposium on Network and Distributed System Security (NDSS 2000), pages 119128. Internet Society, 2000. 